\title{
Aplicação de uma Metodologia de Controle de Nível PI para Reduzir os Efeitos do Fluxo com Golfadas na Produção de Petróleo
}

A. SAUSEN 1 , P.S. SAUSEN ${ }^{2}$, Departamento de Física, Estatística e Matemática, DeFEM, Departamento de Tecnologia, DeTec, Universidade Regional do Noroeste do Estado do Rio Grande do Sul, UNIJUÍ, 98700-000, Ijuí, RS, Brasil.

\begin{abstract}
Resumo. Neste artigo é apresentada a utilização do modelo dinâmico para uma tubulação-separador sob regime de fluxo com golfadas considerando uma aplicação da estratégia de controle de nível Proporcional Integral (PI), cuja finalidade é reduzir os efeitos das golfadas na indústria de produção de petróleo. O modelo utilizado é formado por um sistema de 5 (cinco) Equações Diferenciais Ordinárias (EDOs), não-lineares, acopladas, 6 (seis) parâmetros de sintonia e mais de 40 (quarenta) equações internas, geométricas e de transporte. A aplicação da estratégia de controle de nível PI é realizada considerando a metodologia de controle por bandas, objetivando o amortecimento das vazões de carga oscilatórias que ocorrem em separadores de produção, devido ao regime de fluxo com golfadas. Esta estratégia é comparada com a estratégia de controle de nível PI convencional, amplamente utilizada em processos industriais. A partir da análise dos resultados das simulações verificou-se que a estratégia de controle PI por bandas reduziu as oscilações de vazão ocasionadas pelas golfadas, quando comparada com a estratégia de controle PI convencional.
\end{abstract}

Palavras-chave. Modelagem Matemática, Golfadas, Estratégias de Controle.

\section{Introdução}

Em sistemas de produção de petróleo um fluxo multifásico é transportado, através de linhas de fluxo (i.e., tubulações), dos poços no fundo do oceano até as plataformas de produção na superfície. O fluxo multifásico é composto por uma mistura de água, gás, óleo e impurezas sujeito a formar, devido às características do escoamento, o regime de fluxo com golfadas, que caracteriza-se por fluxo severo e irregular com oscilações de pressão e vazão nos equipamentos do processo, tais como: reservatórios, separadores, compressores, bombas de exportação, entre outros, causando um impacto negativo no sistema de produção de petróleo, com perdas econômicas significativas.

Neste trabalho será abordada a golfada na tubulação ascendente que combinada ou iniciada com a golfada em terrenos é uma das instabilidades mais preocupantes

\footnotetext{
${ }^{1}$ airam@unijui.edu.br.

${ }^{2}$ sausen@unijui.edu.br.
} 
em sistemas tubulação-separador na produção de petróleo $[12,13,14]$. É apresentado na Figura 1 o comportamento cíclico da golfada na tubulação ascendente, que é dividido em quatro fases: (1) formação, a golfada inicia-se com baixa pressão e velocidade dos fluidos na seção de alimentação da tubulação, então em um determinado momento o líquido bloqueia o fluxo de gás no ponto-baixo iniciando a formação de uma golfada de líquido contínua na tubulação ascendente; (2) produção, enquanto a quantidade de líquido na tubulação ascendente aumenta mais rapidamente que a variação da pressão entre o topo e a seção de alimentação, a golfada continua crescendo; (3) explosão, ocorre quando a pressão do gás na seção de alimentação torna-se maior que o peso da coluna de líquido na tubulação ascendente, consequentemente a quantidade de líquido presente na tubulação é enviada para fora do sistema; (4) retorno, após uma grande quantidade de líquido e de gás deixarem o sistema, a pressão na seção de alimentação diminui, então o líquido começa a acumular no ponto-baixo reiniciando o ciclo.

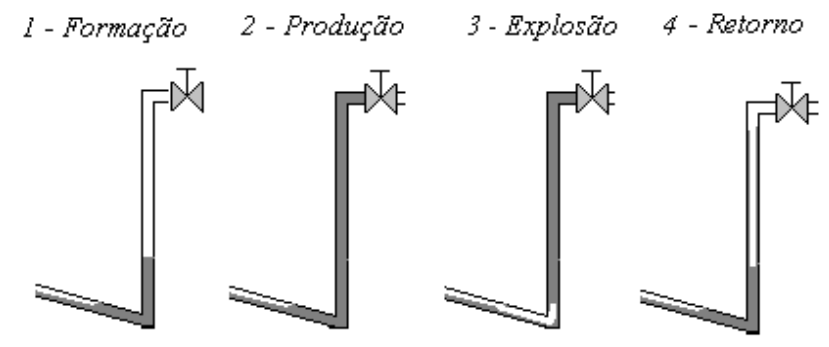

Figura 1: Descrição do ciclo da golfada na tubulação ascendente.

É importante destacar que a propagação da golfada gera diversos problemas indesejados na produção de petróleo, como por exemplo: alterações de períodos com nenhuma produção de líquido, seguido de períodos com grande produção de líquido dentro do separador, o que pode ocasionar redução na capacidade de produção, ineficiência da separação dos componentes da mistura, problemas nos trens de compressão, em alguns casos, quando muito severa, pode gerar parada de emergência na plataforma em decorrência do alto nível de líquido nos separadores $[3,4,6]$.

Neste contexto, novas estratégias que possibilitam evitar a golfada na tubulação, ou minimizar seus efeitos nas unidades de separação passam a ter uma significativa importância. Na literatura, estratégias de controle com realimentação têm-se destacado como uma solução promissora para o tratamento do problema da golfada, pois apresentam desempenho eficiente e econômico, por aumentar a capacidade de produção com reduzido custo de implementação, especialmente quando comparada com as demais $[1,4,5,6,7,8,9,12,13,14]$.

Uma alternativa à aplicação de estratégias de controle consiste em fazer uso de um modelo matemático que descreva adequadamente o processo. Em [9, 11] é apresentado o modelo dinâmico para um sistema tubulação-separador sob regime de fluxo com golfadas formado por um sistema de 5 (cinco) Equações Diferenciais Ordinárias (EDOs), não-lineares, acopladas, 6 (seis) parâmetros empíricos e mais de 40 (quarenta) equações internas, geométricas e de transporte, no presente artigo este modelo será denominado modelo de Sausen. Destaca-se que para a realização das simulações e aplicação de estratégias de controle com realimentação no modelo 
de Sausen, inicialmente, é necessário realizar a sintonia do mesmo, isto é, o cálculo dos seus parâmetros empíricos. Para este procedimento devem ser utilizados dados de um estudo de caso, que podem ser obtidos a partir de uma planta experimental, de um simulador comercial, ou de um processo real. Os dados considerados neste trabalho são obtidos do estudo de caso realizado por $[12,13,14,15]$ para a tubulação, e $[7,8]$ para o separador.

Após a realização da sintonia do modelo de Sausen $[9,11]$ ressalta-se que é importante verificar o quanto as principais variáveis do modelo, tais como, fluxos mássicos de líquido e de gás, pressões na tubulação e no separador, entre outras, variam de comportamento considerando uma variação nos seus parâmetros de sintonia, esta análise é denominada na literatura de análise da sensibilidade [2], cujo objetivo é verificar o efeito das variações dos parâmetros de sintonia sobre os resultados obtidos pela aplicação do modelo. Em $[9,10]$ esta análise é realizada sendo verificado que o modelo é altamente sensível a variação dos seus parâmetros de sintonia, e quando devidamente sintonizado consiste em um ambiente adequado para testar diferentes estratégias de controle com realimentação, no problema da golfada, na produção de petróleo.

Portanto, como parte das estratégias de controle que podem ser utilizadas para evitar ou minimizar a golfada e seus efeitos na indústria de produção de petróleo, neste artigo, é apresentada a aplicação da estratégia de controle de nível Proporcional Integral (PI), na metodologia por bandas, cujo objetivo é amortecer as vazões de carga (i.e., entrada) oscilatórias, ocasionadas pelo regime de fluxo com golfadas, para os equipamentos a jusante do separador. Tal estratégia é comparada com a estratégia de controle de nível PI convencional, amplamente utilizada em processos industriais [5]. Em ambas implementações utiliza-se o modelo de Sausen para representar o processo.

O restante deste artigo está organizado como segue. Na Seção 2. são apresentadas as equações do modelo de Sausen e resultados de simulações. Na Seção 3. são descritas as estratégias de controle de nível PI por bandas, e PI convencional. Na Seção 4. são apresentados os resultados das simulações e sua análise. E por fim, na Seção 5. são apresentadas as conclusões.

\section{O Modelo}

Nesta seção são apresentadas as principais equações do modelo de Sausen para o sistema tubulação-separador apresentado na Figura 2. O modelo é formado por um sistema de 5 (cinco) EDOs, não-lineares, acopladas, baseadas nas equações de conservação de massa do sistema. As hipóteses de modelagem em conjunto com o detalhamento das equações do modelo podem ser encontradas em $[9,10,11]$. A seguir as equações (2.1)-(2.3) descrevem a dinâmica da tubulação, e as equações (2.4)-(2.5) descrevem a dinâmica do separador

$$
\begin{gathered}
\dot{m}_{L}(t)=m_{L, \text { in }}-m_{L, \text { out }}(t), \\
\dot{m}_{G 1}(t)=m_{G, \text { in }}-m_{\text {Gint }}(t), \\
\dot{m}_{G 2}(t)=m_{G 1}(t)-m_{G, \text { out }}(t),
\end{gathered}
$$




$$
\begin{gathered}
\dot{N}(t)=\frac{\sqrt{r_{s}^{2}-\left(r_{s}-N(t)\right)^{2}}}{2 H_{4} \rho_{L} N(t)\left[3 r_{s}-2 N(t)\right]}\left[m_{L, \text { out }}(t)-m_{L S, \text { out }}(t)\right], \\
\dot{P}_{G 1}(t)=\frac{\left\{\rho_{L} \Phi\left[m_{G, \text { out }}(t)-m_{G S, \text { out }}(t)\right]+P_{G 1}(t)\left[m_{L, \text { out }}(t)-m_{L S, \text { out }}(t)\right]\right\}}{\rho_{L}\left[V_{S}-V_{L S}(t)\right]},
\end{gathered}
$$

onde $m_{L}(t)$ é a massa de líquido no ponto-baixo da tubulação, em $k g ; m_{G 1}(t)$ é a massa de gás na seção de alimentação da tubulação, em $k g ; m_{G 2}(t)$ é a massa de gás no topo da tubulação ascendente, em $k g ; N(t)$ é o nível de líquido dentro do separador, em $m ; P_{G 1}(t)$ é a pressão do gás dentro do separador, em $N / m^{2} ; \dot{m}_{L}(t)$, $\dot{m}_{G 1}(t), \dot{m}_{G 2}(t), \dot{N}(t)$, e $\dot{P}_{G 1}(t)$ são respectivamente suas derivadas em relação ao tempo; $m_{L, \text { in }}$ e $m_{G, \text { in }}$ são os fluxos mássicos de líquido e gás que entram na seção de alimentação da tubulação, considerados constantes, em $\mathrm{kg} / \mathrm{s} ; m_{L, \text { out }}(t)$ e $m_{G \text {, out }}(t)$ são os fluxos mássicos de líquido e gás que saem através da válvula 1 no topo da tubulação ascendente e por conseguinte entram no separador, em $\mathrm{kg} / \mathrm{s} ; m_{\text {Gint }}(t)$ é o fluxo mássico de gás interno na tubulação que flui da seção de alimentação para o topo da tubulação ascendente, em $\mathrm{kg} / \mathrm{s} ; m_{L S, \text { out }}(t)$ é o fluxo mássico de líquido que sai através da válvula 2 do separador, em $\mathrm{kg} / \mathrm{s} ; m_{G S \text {, out }}(t)$ é o fluxo mássico de gás que sai através da válvula 3 do separador, em $\mathrm{kg} / \mathrm{s} ; r_{s}$ é o raio do separador, em $m ; C$ é o comprimento do separador, em $m ; \rho_{L}$ é a densidade de líquido no sistema, em $\mathrm{kg} / \mathrm{m}^{3} ; V_{S}$ é o volume total do separador, em $\mathrm{m}^{3} ; V_{L S}(t)$ é o volume de líquido no separador, em $m^{3} ; \Phi=\frac{R T}{M_{G}}$ é uma constante composta por $R$ constante universal do gases ideais $\left(8314 \frac{J}{K . k m o l}\right), T$ temperatura no sistema, em $K$; e $M_{G}$ peso molecular do gás, em $\mathrm{kg} / \mathrm{kmol}$.

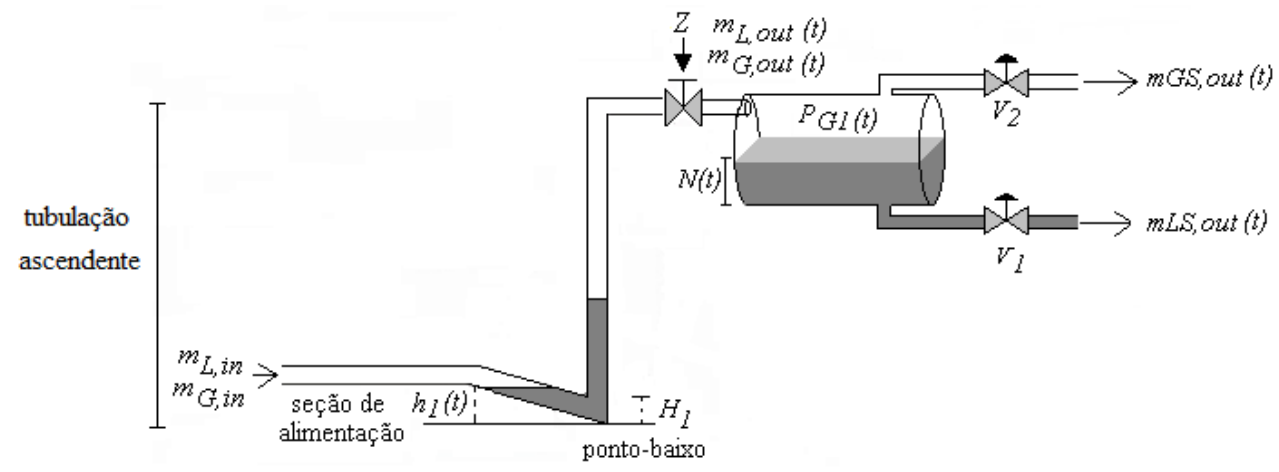

Figura 2: Ilustração de um sistema tubulação-separador com a golfada em formação.

O fluxo mássico da mistura $m_{m i x, o u t}(t)$, composta de líquido e gás, que sai da tubulação para o separador é determinado através da equação simplificada da válvula $Z$ no topo da tubulação ascendente, dada por

$$
m_{\text {mix }, \text { out }}(t)=z K_{1} \sqrt{\rho_{T}(t)\left(P_{2}(t)-P_{G 1}(t)\right)},
$$

onde $z$ é a abertura da válvula $(0-100 \%) ; K_{1}$ é um parâmetro de sintonia do modelo; $\rho_{T}(t)$ é a densidade na válvula em $\mathrm{kg} / \mathrm{m}^{3} ; P_{2}(t)$ é a pressão no topo da tubulação ascendente em $N / m^{2}$. 
Considerando a equação (2.6) é possível obter respectivamente o fluxo mássico de líquido

$$
m_{L, \text { out }}(t)=\alpha_{L}^{m}(t) m_{\text {mix }, \text { out }}(t)
$$

e o fluxo mássico de gás

$$
m_{G, \text { out }}(t)=\left[1-\alpha_{L}^{m}(t)\right] m_{m i x, \text { out }}(t),
$$

onde $\alpha_{L}^{m}(t)$ é fração de líquido na válvula.

O fluxo mássico de líquido e de gás que saem do separador são representados, respectivamente, pela equação da válvula $V_{1}$ de líquido

$$
m_{L S, \text { out }}(t)=z_{L} K_{4} \sqrt{\rho_{L}\left[P_{G 1}(t)+g \rho_{L} N(t)-P_{O L 2}\right]},
$$

onde $z_{L}$ é a abertura da válvula $(0-100 \%) ; K_{4}$ é um parâmetro de sintonia do modelo; $g$ é a gravidade; $P_{O L 2}$ é a pressão a jusante da válvula de líquido, em $N / m^{2}$ e considerada constante; e pela equação da válvula $V_{2}$ de gás, dada por

$$
m_{G S, \text { out }}(t)=z_{G} K_{5} \sqrt{\rho_{G}(t)\left[P_{G 1}(t)-P_{G 2}\right]},
$$

onde $z_{G}$ é a abertura da válvula $(0-100 \%) ; K_{5}$ é um parâmetro de sintonia do modelo; $\rho_{G}(t)$ é a densidade do gás, em $\mathrm{kg} / \mathrm{m}^{3} ; P_{G 2}$ é a pressão a jusante da válvula de gás, em $N / m^{2}$ e considerada constante.

Os fluxos mássicos de líquido e gás que entram na seção de alimentação da tubulação (i.e., $m_{L, i n}$ e $m_{G, i n}$ ) são consideradas perturbações do processo e podem ser constantes, ou dependentes da pressão, neste trabalho são assumidos constantes. A seguir são apresentadas as equações que descrevem o deslocamento de gás e líquido dentro da tubulação.

\subsection{Deslocamento de gás na tubulação}

O deslocamento de gás ocorre através de uma relação entre o fluxo mássico de gás e a variação de pressão dentro da tubulação. Os dois principais parâmetros que determinam o deslocamento e a velocidade do gás no sistema são a variação de pressão $\Delta P(t)=P_{2}(t)-P_{1}(t)$ na tubulação, e a área livre no ponto-baixo dado pelo nível de líquido relativo $f\left(h_{1}(t)\right)=\left(H_{1}-h_{1}(t)\right) / H_{1}$. Logo, a equação que representa o deslocamento de gás, quando $h_{1}(t)<H_{1}$, é dada por

$$
v_{G 1}(t)=K_{2} f\left(h_{1}(t)\right) \sqrt{\frac{P_{1}(t)-P_{2}(t)-g \rho_{L} \alpha_{L}(t) H_{2}}{\rho_{G 1}(t)}},
$$

onde $K_{2}$ é um parâmetro de sintonia do modelo, $P_{1}(t)$ é a pressão na seção de alimentação do encanamento, $\mathrm{N} / \mathrm{m}^{2} ; h_{1}(t)$ é o nível de líquido no ponto-baixo, $m ; H_{1}$ é o diâmetro no ponto-baixo da tubulação, $m ; H_{2}$ é a altura da tubulação ascendente, $m$. Por outro lado, se $h_{1}(t) \geq H_{1}$ a velocidade de gás no ponto-baixo é igual a zero. 


\subsection{Deslocamento de líquido na tubulação}

A distribuição de líquido no sistema ocorre através de uma equação de carregamento, ou seja, considera-se que o gás carrega o líquido pela tubulação ascendente, então modela-se diretamente a fração de volume de líquido $\alpha_{L T}(t)$ que sai através da válvula $Z$ no topo da tubulação ascendente. É assumido que esta transição depende de um parâmetro $q(t)$, logo o carregamento de líquido é representado pela equação dada por

$$
\alpha_{L T}(t)=\alpha_{L T}^{*}(t)+\frac{q^{n}(t)}{1+q^{n}(t)}\left(\alpha_{L}(t)-\alpha_{L T}^{*}(t)\right),
$$

onde

$$
q(t)=\frac{K_{3} \rho_{G 1}(t) v_{G 1}^{2}(t)}{\rho_{L}-\rho_{G 1}(t)}
$$

e $K_{3}$ e $n$ são parâmetros de sintonia do modelo.

Os detalhes da modelagem matemática do modelo de Sausen podem ser encontrados em $[9,11]$, e suas equações interna, de transporte e geométricas podem ser encontradas em $[9,14]$.

\subsection{Resultados das simulações do modelo de Sausen}

Nesta seção são apresentados os resultados das simulações do modelo de Sausen. Inicialmente são calculados os parâmetros de sintonia, ou seja, $K_{1}, K_{2}, K_{3}, n, K_{4}$, e $K_{5}$, cujos resultados são apresentados na Tabela 1. Para a realização dos cálculos são necessários dados de um sistema real ou de uma malha experimental, porém um conjunto completo destes dados não são encontrados na literatura, e também não são fornecido pelas indústrias de produção de petróleo. Para contornar este problema neste trabalho é utilizado um estudo de caso realizado por [14] através do simulador de fluxo multifásico OLGA para a tubulação sob golfadas, em conjunto com os dados de um separador dimensionado a partir dos trabalhos de $[7,8]$.

Tabela 1: Parâmetros de sintonia para o modelo dinâmico de um sistema tubulaçãoseparador.

\begin{tabular}{|c|c|c|c|c|c|}
\hline$\varphi$ & $K_{1}$ & $K_{2}$ & $K_{3}$ & $K_{4}$ & $K_{5}$ \\
\hline 2,55 & 0,005 & 0,8619 & 1,2039 & 0,002 & 0,0003 \\
\hline
\end{tabular}

A seguir são apresentados os resultados das simulações considerando a abertura da válvula $\mathrm{Z}$ no topo da tubulação ascendente em $50 \%$. Na Figura 3 são apresentadas as oscilações de pressão na seção de alimentação do encanamento $P_{1}(t)$ e no topo da tubulação $P_{2}(t)$. Na Figura 4 é mostrada a dinâmica dos fluxos mássicos de líquido e gás que entram (esquerda) e saem (direita) do separador. Observa-se que o regime de fluxo é oscilatório durante todo o intervalo de simulação caracterizando o regime de fluxo com golfadas no sistema. Na Figura 5 são apresentados o nível de líquido (esquerda) e a pressão do gás (direita) dentro do separador observa-se que a golfada acontece entre um intervalo de tempo de aproximadamente de $12 \mathrm{~min}$ dentro do vaso. 


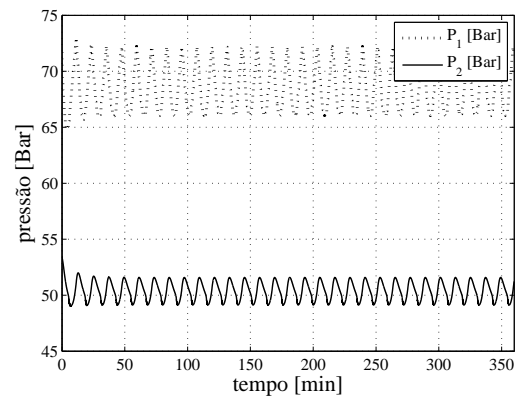

Figura 3: Pressões no sistema tubulação-separador com golfadas.
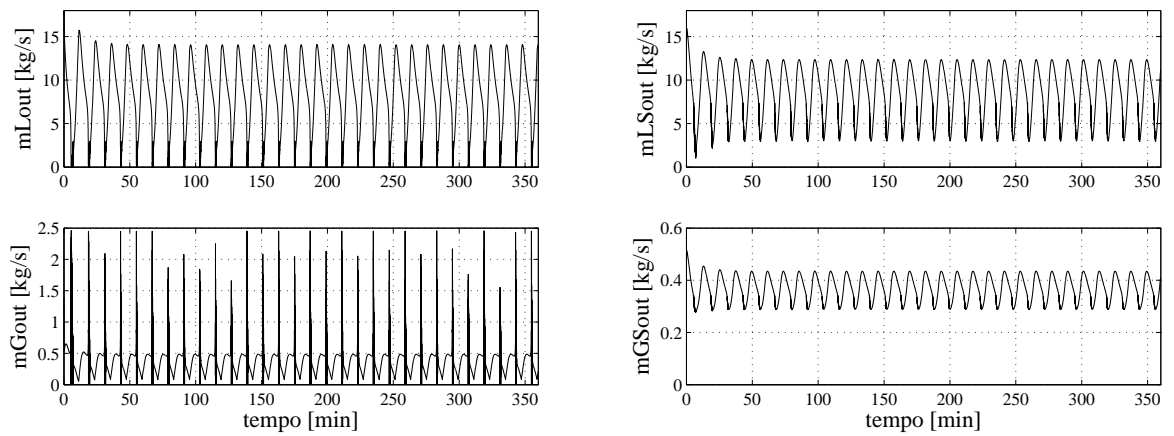

Figura 4: (Esquerda) Fluxos mássicos de gás e líquido que entram no separador. (Direita) Fluxos mássicos de gás e líquido que saem do separador.
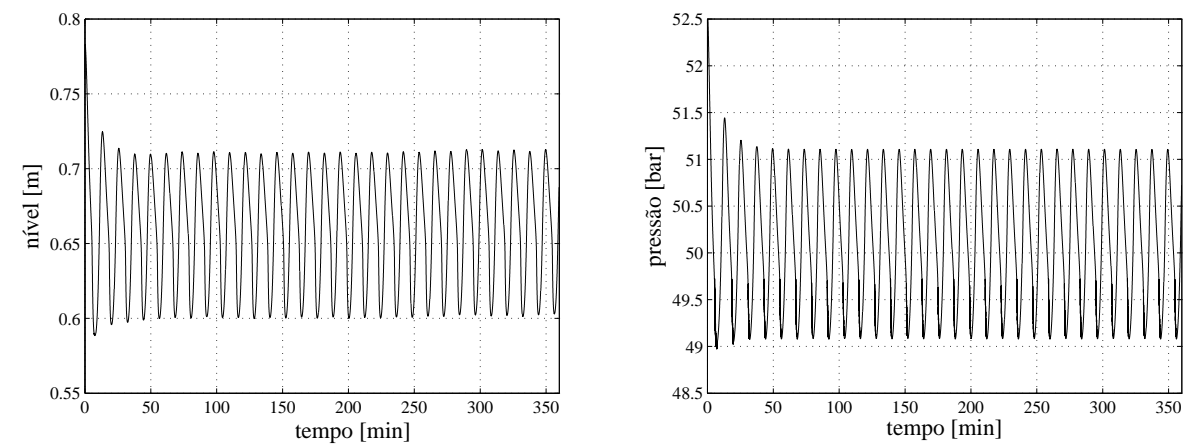

Figura 5: (Esquerda) Nível de líquido no separador. (Direita) Pressão do gás no separador.

\section{Estratégias de Controle}

$\mathrm{Na}$ indústria de produção de petróleo são utilizados controladores do tipo PI para controlar as variações do nível de líquido, ocasionadas pelo regime de fluxo com golfadas, nos separadores de produção. Porém, não é aconselhável a aplicação da estratégia de controle de nível convencional que utiliza o nível (i.e., variável controlada) fixo no setpoint, pois as oscilações provenientes do regime de fluxo com 
golfadas são transferidas para a saída causando perturbações nos equipamentos do processo [3, 5]. Por outro lado, em [7, 8] é definida uma metodologia de controle de nível denominada controle por bandas, onde é proposto que durante a atuação do controlador o nível pode variar entre o máximo e o mínimo de uma banda, conforme a Figura 6, de modo que as vazões de saída estejam próximas ao valor médio da vazão de entrada. A adoção desta estratégia dispensa medições de vazão, podendo ser aplicada em qualquer vaso de acúmulo.

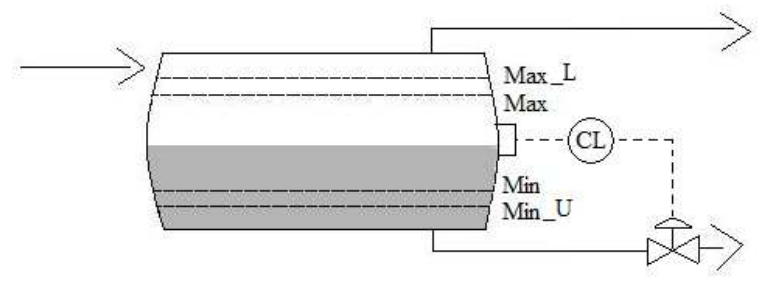

Figura 6: Esquema de um controlador de nível (CL), considerando a metodologia por bandas, aplicado em separador cilíndrico horizontal bifásico.

No controle por bandas, quando o nível encontra-se dentro da banda, utiliza-se a média móvel da ação de controle de um controlador PI lento, pois reduzindo a capacidade de atuação do controlador obtém-se uma maior flutuação do nível de líquido dentro do separador. A média móvel é calculada ao longo de um determinado intervalo de tempo, este intervalo deve ser maior que o período $T$ da golfada. Nos casos em que os limites da banda são ultrapassados, comuta-se a ação de controle em média móvel do controlador PI lento, para um controlador PI de ação rápida por determinado tempo, visando garantir o retorno do nível para dentro da banda e, em caso positivo, a ação de controle volta a ser em média móvel. De modo a evitar variações bruscas na ação de controle durante a comutação entre os modos de operação dentro da banda e fora da banda, é sugerido usar a média entre as ações de controle PI e em média móvel [7, 8].

Neste contexto, no presente artigo é realizada a aplicação da estratégia de controle de nível PI no modelo de Sausen considerando 2 (duas) metodologias: (1) controle de nível PI convencional, ou seja, o nível deve permanecer fixo no setpoint (i.e., referência); (2) controle de nível PI por bandas [7], ou seja, o nível pode flutuar em torno do setpoint considerando dois limites dentro do vaso (i.e., um inferior e um superior). Para estas implementações é usado o algoritmo em velocidade do controlador PI [5], cuja equação é dada por

$$
\Delta u(t)=k_{p} \Delta e(t)+k_{p} \frac{1}{T_{i}} T_{a} e(t),
$$

onde $\Delta u(t)$ é a variação da ação de controle; $k_{p}$ é o ganho do controlador; $\Delta e(t)$ é a variação do erro de rastreamento do processo; e $T_{a}$ é o período de amostragem do controlador.

Considera-se que a dinâmica da válvula, isto é, o tempo para que sua abertura alcance o valor da ação de controle é curto, portanto, isto implica que a abertura da mesma pode ser considerada a própria ação de controle. Conforme a equação (3.1), na ação de controle proporcional a ação de correção na válvula é proporcional 
a variação do erro de rastreamento $\Delta e(t)$, onde $e(t)$ é o desvio entre a variável controlada (i.e, nível de líquido) em relação ao setpoint (i.e., nível de referência), e a ação integral vai atuar no processo enquanto houver diferença entre o valor medido (nível) e o valor desejado (setpoint) [5].

\section{Análise e Resultados das Simulações}

Nesta seção são apresentados os resultados das simulações realizadas na ferramenta computacional Matlab utilizando o modelo de Sausen $[9,10,11]$. Para a implementação do controle por bandas, neste trabalho, foi utilizado um separador que possui comprimento de $4,5 \mathrm{~m}$ e diâmetro de $1,5 \mathrm{~m}$, seguindo os padrões utilizado nos trabalhos de [7, 8], o qual aplica este controle na plataforma P-07 da Petrobrás. O setpoint considerado é $0,75 \mathrm{~m}$ (i.e., metade do separador) e a banda é $0,2 \mathrm{~m}$, onde o nível máximo de líquido permitido passou a ser $0,95 \mathrm{~m}$ e o nível mínimo 0,55 m. A banda foi definida seguindo os trabalhos de $[3,7,8]$.

Para a simulação da estratégia de controle PI de nível convencional os valores utilizados para as constantes $k_{p}$ e $T_{i}$ do controlador são respectivamente 10 e 1380 $s$, conforme a metodologia denominada método heurístico de sintonia para controladores de nível $[4,5]$. Na estratégia de controle PI por bandas o nível pôde flutuar livremente dentro dos limites da banda no vaso, neste caso, para o controlador PI de ação lenta (i.e., atuação dentro da banda) utilizou-se $k_{p}=0,001$ e $T_{i}=100000 \mathrm{~s}$, para o controlador PI de ação rápida (i.e., atuação fora da banda) usou-se $k_{p}=0,1$ e $T_{i}=1000 \mathrm{~s}$, o período para o cálculo da média móvel do controlador PI foi de $T=1000 \mathrm{~s}$. A seguir são apresentados os resultados das simulações para abertura da válvula no topo da tubulação ascendente fixa em $z=20 \%$.
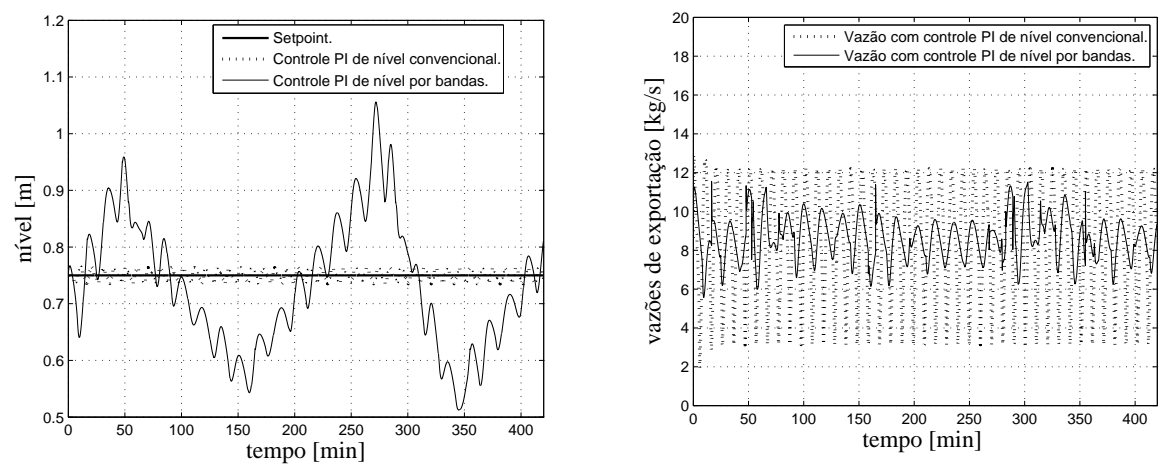

Figura 7: (Esquerda)Variações do nível de líquido dentro do separador considerando. (Direita) Variações da vazão de saída de líquido do separador. Abertura da válvula $z$ fixa em $20 \%$.

Na Figura 7 são apresentados os resultados das simulações (esquerda) das variações do nível de líquido dentro do separador com a aplicação do controle PI de nível convencional (linha pontilhada) e com a aplicação do controle PI de nível por bandas (linha sólida), também são mostradas (direita) as respectivas vazões de exportação (i.e., saída) de líquido do vaso de separação. 
Ainda com o objetivo de avaliar a estratégia de controle de nível PI por bandas utiliza-se a abertura $z$ da válvula no topo da tubulação ascendente, variável, isto é, $z=20 \%, z=25 \%, z=30 \%$ e $z=35 \%$. Sendo assim, na Figura 8 são apresentadas (esquerda) as variações do nível de líquido dentro do separador com a aplicação do controle PI de nível convencional (linha pontilhada), e com a aplicação do controle PI de nível por bandas (linha sólida), também são mostradas (direita) as respectivas vazões de exportação (i.e., saída) de líquido do vaso de separação.
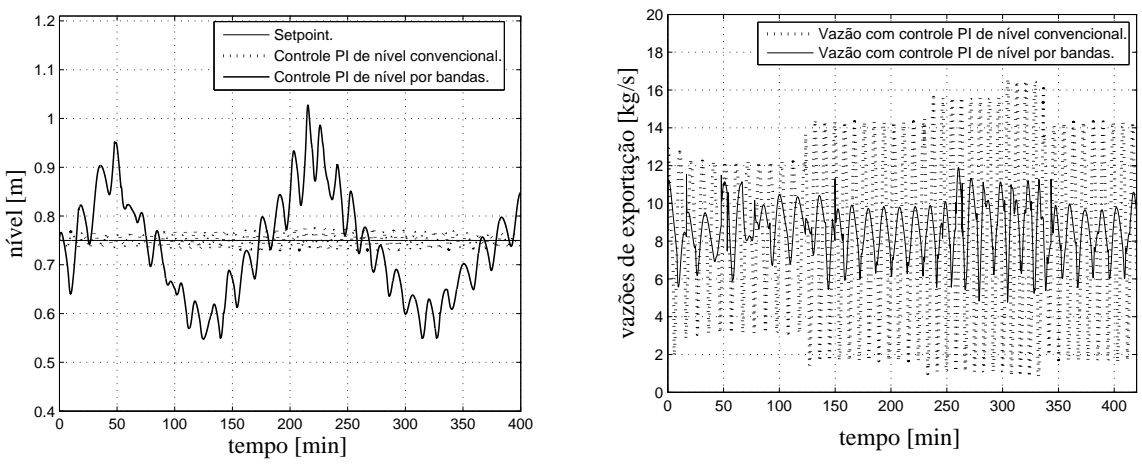

Figura 8: (Esquerda)Variações do nível de líquido dentro do separador. (Direita) Variações da vazão de saída de líquido do separador. Abertura da válvula $z$ variável

A partir dos resultados das simulações é observado que com a estratégia de controle PI de nível convencional as vazões de carga (i.e., entrada) são transferidas para a saída do separador, conforme pode ser verificado nas Figuras 7 e 8 (i.e., linha pontilhada, a direita) ocasionando perturbações nos equipamentos à jusante do processo. Por outro lado, considerando a estratégia de controle PI de nível por bandas é possível reduzir as oscilações das vazões de exportação, conforme observado nas Figuras 7 e 8 (i.e., linha sólida, a direita), a partir das flutuações do nível de líquido dentro do vaso, de acordo com as Figuras 7 e 8 (i.e., linha sólida, a esquerda).

\section{Conclusões}

O regime de fluxo com golfadas ocasiona vazões de carga e exportação oscilatórias em plataformas de produção de petróleo, causando perturbações nos equipamentos do processo, e prejuízos de ordem econômica significativos. Com o objetivo de reduzir as variações de vazão de exportação foi implementada, neste trabalho, uma metodologia de controle de nível denominada controle de nível PI por bandas, onde é permitido que o nível flutue dentro de uma banda de modo que as vazões de exportação sejam o menos oscilatórias possíveis. Em seguida, esta estratégia foi comparada com a estratégia de controle de nível PI convencional. A partir dos resultados das simulações observou-se uma significativa redução nas oscilações das vazões de exportação do vaso com a aplicação da estratégia de controle de nível PI por bandas, quando comparada com a aplicação da estratégia de controle de nível PI convencional. 
Abstract. This paper presents the use of the dynamic model for a pipelineseparator system under the slug flow regime considering an application of level control Proportional Integral (PI), whose purpose is reduces the effects of the slug flow in the oil industry. The model used has 5 (five) Ordinary Differential Equations (ODEs), coupled, nonlinear, 6 (six) tuning parameters and more than 40 (forty) internal, geometric and transport equations. The application of the level control's strategy PI is performed thought the methodology for bands, whose objective is the damping of the liquid mass flowrate that enters in production's separators due to slug flow regime. This strategy is compared with the level control's strategy PI conventional, widely used in industrial processes. The simulation results of the level control's strategy PI thought the methodology for bands shows that the oscillations of the liquid mass flowrate caused by slug flow were reduced, when compared with the level control's strategy PI conventional.

\section{Referências}

[1] A.M. Borges Filho, M.A.A. de Meira, C.E.M. Vaz, Sistema de controle de golfadas, em "Rio Oil e Gas Expo and Conference 2004", Rio de Janeiro, 2004.

[2] D.G. Cacuci, "Sensitivity and Uncertainty Analysis Theory", Chapman and Hall/CRC, New York, 2003.

[3] M.C.M. de Campos, L.A. Costa, A.E. Torres, D.C. Schmidt, Controle Avançado dos Níveis dos Separadores de Plataformas de Produção, em "1 ${ }^{\circ}$ CICAP Congresso de Instrumentação, Controle e Automação da Petrobrás (I CICAP)", $\mathrm{RJ}$,

[4] M.C.M. Campos, P. Laureiro, A.M. Borges Filho, Novas Estratégias de Controle para a Plataforma de Petróleo P-55, em "Rio Oil e Gas Expo and Conference 2006", Rio de Janeiro, 2006.

[5] M.C.M. de Campos, H.C.G. Teixeira, "Controles Típicos de Equipamentos e Processos Industriais", Editora Edgard Blücher, Rio de Janeiro, 2006.

[6] M.J. Godhavn, F.P. Mehrdad, P. Fuchs, New slug control strategies, tuning rules and experimental results, Journal of Process Control, 15 (2005), 547-577.

[7] G.C. Nunes, Controle por bandas: conceitos básicos e aplicação no amortecimento de oscilações de carga em unidades de produção de petróleo, em "Petróleo Centro de Pesquisas (Cenpes)", pp. 151-165, Rio de Janeiro, 2004.

[8] G.C. Nunes, Controle por bandas para processamento primário: aplicação e conceitos fundamentais, em "Boletim Técnico Petrobrás (Cenpes)", Rio de Janeiro, 2004 .

[9] A. Sausen, "Modelagem Matemática de um Sistema Tubulação-Separador sob Regime de Fluxo com Golfadas e Controle de Nível Considerando um Algoritmo de Erro-Quadrático", Tese de Doutorado, Universidade Federal de Campina Grande-UFCG, Brasil, 2009. 
[10] A. Sausen, Análise da sensibilidade do modelo dinâmico simplificado para uma tubulação-separador sob golfadas, TEMA - Tendências em Matemática Aplicada e Computacional, 11, No 3 (2010), 245-256.

[11] A. Sausen, P.R. Barros, Modelo dinâmico simplificado para um sistema encanamento-riser-separador sob regime de fluxo com golfadas, em "Seleta do Dincon 2008" (M. Meneguette Jr. et al., eds.), TEMA - Tendências em Matemática Aplicada e Computacional, Vol. 9, pp. 341-350, SBMAC, 2008.

[12] E. Storkaas, S. Skogestad, J.M. Godhavn, A low-dimensional dynamic model of severe slugging for control design and analysis, in "11th International Conference on Multiphase flow (Multiphase03)", pp. 117-133, San Remo, Italy, 2003.

[13] E. Storkaas, S. Skogestad, J.M. Godhavn, Stabilization of severe slugging based on a low-dimensional nonlinear model, in "AIChE Annual Meeting", Paper 259e, Indianapolis, USA, 2002.

[14] E. Storkaas, "Stabilizing Control and Controllability: Control Solutions to Avoid Slug Flow in Pipeline-Riser Systems", Tese de Doutorado, Norwegian University of Science and Technology, Norwegian, 2005.

[15] Y. Taitel, F.E. Jansen, O. Shohan, The elimination of severe sluggingexperiments and modeling, International Journal of Multiphase Flow, 22 (1996), 1055-1072. 